\title{
Winners and Losers: Allocating Scare Resources for Patients with Hepatocellular Carcinoma
}

\author{
Kenneth Washburn and Sylvester Black \\ Division of Transplant Surgery, Department of Surgery, The Ohio State University Wexner Medical Center, Columbus, $\mathrm{OH}$
}

The allocation of a scare resource requires considerable thought and application of ethical principles. This is a constant struggle as we try and balance the principles of population utility versus individual justice in the field of organ transplantation. Scarce donor organs, in this case, livers, are distributed according the illness of the patient. In many countries, this is according to the MELD score, which reflects the 90-day mortality for patients with endstage liver disease. In most situations patients with hepatocellular cancer (HCC) do not manifest a high enough native MELD score to be competitive for a transplant and must be given exception points to increase their chances of transplant. Too much priority and non-HCC patients have a more challenging time receiving a transplant. Too little and HCC patients start dropping off the waitlist due to disease progression beyond reasonable criteria. For many years in the United States, these HCC patients have received too much priority despite numerous policy changes. This has been shown multiple times when one looks at the dropout rate from the liver transplant waiting list. ${ }^{1,2}$ Recent policy changes in the United States have changed the waiting time and exception points that HCC patients will receive in attempts to place these patients more equitably with nonHCC patients awaiting a liver transplant (LT). Only patients meeting strict criteria are eligible for excess priority for transplant. The criteria (Milan) have been challenged as being too restrictive, and thus many series have been reported showing good results with patients with HCC beyond Milan. ${ }^{3}$

(C) Society of Surgical Oncology 2017

First Received: 24 June 2017;

Published Online: 7 July 2017

K. Washburn

e-mail: ken.washburn@osumc.edu
In the article by Grat et al., the authors show that the 5 year outcome of patients with more advanced HCC have comparable survivals to the sickest patients (MELD > 40). ${ }^{4}$ Based on these observations, they argue that more liberal criteria regarding LT should be employed for patients with more advanced HCC. There is no doubt that some patients with more advanced-staged HCC disease can benefit from transplantation. In this series, 5-year patient survival for those patients with high-risk HCC was 55\% compared with $56 \%$ for non-HCC high-risk patients (MELD $>40$ ). However, it is important to consider patient selection in this process. Those at the highest MELD scores are very carefully selected for LT. It is not clear how patients with more advanced HCC are selected for LT in this report. Response to therapy is an important selection factor in patients with more advanced disease, and it is not clear whether this was used as a discriminating variable in patient selection. The authors identify preoperative factors of AFP, tumor size, or volume as strong predictors of disease recurrence, which have been reported many times previously. Given the comparable 5-year survival for the sickest patients and the highest-risk HCC patients, the authors advocate for expanding criteria to these high risk HCC patients.

There is no doubt that patients with many different disease etiologies can benefit from LT when the alternative is death if a transplant is not received. The comparison of two high-risk groups with markedly worse 5-year survival than the medium- or low-risk groups is to some degree a false comparison. If there were no limitation on donor organs, then this might be a fair comparison; yet that does not exist. It has been shown that the negative impact of expanding selection criteria often offsets the benefits when the 5-year posttransplant survival rate falls below $61 \%$ in the United States. ${ }^{5}$ This observation is dependent of the wait times and MELD score at transplant for non-HCC patients. Transplantation is predicated on the good will of society for the donation of organs for transplant. At least in 
the United States, transplant centers are held to rigorous outcome standards. The imperative is to obtain the most life years out of an organ while preventing as many deaths as possible on the wait list. The authors do admit that the most controversial aspect of increasing access of HCC patients to LT is the impact on the non-HCC patients. However, no solution is proposed to ameliorate this effect. A major limitation to virtually all studies proposing expansion of HCC criteria is the impact the policy change would have on the non-HCC patients. We must remember that there are therapies for patients with HCC that may not cure their disease but can certainly prolong their lives. The same cannot be said for medium-risk patients with a relatively high MELD score $(>30)$. These patients have a very high (50\%) 3-month mortality with very limited options to keep them alive. Thus, we must realize that shifting more scarce organs to high-risk HCC patients certainly would impact negatively the non-HCC patients.

Expansion of the permissible HCC criteria for LT is admirable and probably advisable in certain circumstances as it has been shown that acceptable results can be achieved in selected patient populations. However, we must be mindful of how such a policy change might impact negatively the remaining patients awaiting a donor liver. More precise ways of predicting poor post LT outcomes would be helpful in selecting appropriate patients for expanded HCC criteria. A 5-year outcome of 50\% for high-risk patients (as shown in this study) might be acceptable in selected situations, but in general this is below that of most studies showing outcomes around $70 \%$. Prior work evaluating tumor biology as a discriminating factor has shown acceptable 5-year survival rates of $70 \%$ in patients with
HCC beyond the Milan criteria. ${ }^{6}$ Perhaps using pretransplant biopsies with a continuous scoring system, such as the HALT-HCC, might enable transplant centers to predict more accurately which patients with more advanced HCC might be most appropriate for $\mathrm{LT}^{7}$ Before we jump to expand further the criteria for HCC patients, we must have better selection criteria that allows us to discern more clearly the risk of recurrence and death.

\section{REFERENCES}

1. Washburn K, Edwards E, Harper A, Freeman R. Hepatocellular carcinoma patients are advantaged in the current liver transplant allocation system. Am J Transplant. 2010;10(7):1643-48.

2. Patel MS, Kohn R, Kratz JR, Shah JA, Markmann JF, Vagefi PA. The race to liver transplantation: a comparison of patients with and without hepatocellular carcinoma from listing to post-transplantation. J Am Coll Surg. 2015;220:1001-07.

3. Zaydfudim VM, Vachharajani N, Klintmalm GB, et al. Liver resection and transplantation for patients with hepatocellular carcinoma beyond Milan Criteria. Ann Surg. 2016;264:650-58.

4. Grat M, Stypułkowski J, Patkowski W, et al. Challenging the principle of utility as a barrier for wider use of liver transplantation for hepatocellular cancer. Ann Surg Oncol. 2017 (in press).

5. Volk ML, Vijan S, Marrero JA. A novel model measuring the harm of transplanting hepatocellular carcinoma exceeding Milan criteria. Am J Transplant. 2008;8:839-46.

6. DuBay D, Sandroussi C, Sandhu L, et al. Liver transplantation for advanced hepatocellular carcinoma using poor tumor differentiation on biopsy as an exclusion criterion. Ann Surg. 2011;253: 166-72.

7. Sasaki K, Firl DJ, Hashimoto K, Fujiki M, Diago-Uso T, Quintini C, Eghtesad B, Fung JJ, Aucejo FN, Miller CM. Development and validation of the HALTHCC score to predict mortality in liver transplant recipients with hepatocellular carcinoma: a retrospective cohort analysis. Lancet Gastroenterol Hepatol. 2017. doi:10.1016/ S2468-1253(17)30106-1. 